\title{
Exact slow-fast decomposition of the Hamilton-Jacobi equation of singularly perturbed systems
}

\author{
E. Fridman \\ Dept. of Electrical Eng. - Systems \\ Tel Aviv University, Tel Aviv 69978, Israel \\ e-mail:emilia@eng.tau.ac.il
}

\begin{abstract}
We study a Hamilton-Jacobi partial differential equation, arizing in optimal control problem for an affine nonlinear singularly perturbed system. This equation is solvable iff there exists a special invariant manifold of the corresponding Hamiltonian system. We obtain exact slow-fast decomposition of the Hamiltonian system and of the special invariant manifold into the slow and the fast ones. We get sufficient conditions for the solvability of the HamiltonJacobi equation in terms of the reduced-order slow submanifold, or in the hyperbolic case, in terms of a reduced-order slow Riccati equation. On the basis of this decomposition we construct asymptotic expansions of the optimal state-feedback, optimal trajectory and optimal open-loop control in the powers of a small parameter.
\end{abstract}

\section{Introduction}

Nonlinear optimal control problem for singularly perturbed systems leads to a high dimensional Hamilton-Jacobi partial differential equation of twotime-scales for an optimal controller evaluation [3]. To alleviate the difficulties caused by the high dimensionality and the stiffness that result from the interaction of slow and fast dynamical modes, a composite controller was designed [3], [6]. This $\epsilon$ - independent controller was based on the reducedorder slow and fast subproblems. Also series expansion method has been developed for approximate solution of Hamilton-Jacobi equations. It was shown that truncated expansion satisfies equations to $O\left(\epsilon^{k}\right)$-accuracy. However near-optimality of the approximate solution (in the sense of its closeness to the exact one) has not been studied yet. It was proved in [1] that the leading term in the approximation converges to the exact solution as $\epsilon \rightarrow 0$.

In the linear case high-order numerical approximations were constructed in [10] on the basis of the exact decomposition of the full-order Riccati equation into the reduced-order Riccati and linear algebraic equations. In [4] exact decompostion of the singularly perturbed $H_{\infty}$ Riccati equation was obtained and asymptotic approximation to its solution was constructed.

In the present paper we get the nonlinear counterpart of [10] and [4]. We apply the geometric approach of [5], [7] which relates Hamilton-Jacobi equations with special invariant manifolds of Hamiltonian systems. We obtain the exact decomposition of the special slow-fast manifold into the reducedorder slow submanifold of the Hamiltonian system and the fast manifold of an auxiliary system. We construct a higher-order approximation to the optimal closed-loop and open-loop controls and optimal trajectory in the form of expansion in the powers of $\epsilon$. 


\section{Problem Formulation}

Consider the optimal control problem for the system

$$
\begin{aligned}
& \dot{x}_{1}=a_{1}\left(x_{1}\right)+A_{1}\left(x_{1}\right) x_{2}+B_{1}\left(x_{1}\right) u \\
& \epsilon \dot{x}_{2}=a_{2}\left(x_{1}\right)+A_{2}\left(x_{1}\right) x_{2}+B_{2}\left(x_{1}\right) u
\end{aligned}
$$

with respect to the functional

$J=\int_{0}^{\infty}\left[r\left(x_{1}\right)+s^{\prime}\left(x_{1}\right) x_{2}+x_{2}^{\prime} Q\left(x_{1}\right) x_{2}+u^{\prime} R\left(x_{1}\right) u\right] d t$

where $x_{1}(t) \in \mathbf{R}^{\mathbf{n}_{1}}$ and $x_{2}(t) \in \mathbf{R}^{\mathbf{n}_{2}}$ are the state vectors, $x=\operatorname{col}\left\{x_{1}, x_{2}\right\}, \quad u(t) \in \mathbf{R}^{\mathbf{m}}$ is the control input. Prime denotes the transposition of a matrix. The functions $a_{i}, A_{i}, B_{i}, p, s$ and $Q$ are smooth. We assume also that $a_{i}(0)=0, r(0)=0$ and $s(0)=0$. Assume that $Q \geq 0, R>0$ and $r+s^{\prime} x_{2}+x_{2}^{\prime} Q x_{2} \geq 0$.

The system (2.1) has a standard singularly perturbed form in the sense that it is nonlinear only on the slow variable $x_{1}$ (see e.g.[3,6]). However in the contrast to the standard case, $A_{22}$ is allowed to be singular.

Denote by $|\cdot|$ the Euclidian norm of a vector. Consider the Hamiltonian function

$\mathcal{H}\left(x_{1}, x_{2}, p_{1}, p_{2}\right)=r+s^{\prime} x_{2}+x_{2}^{\prime} Q x_{2}+p_{1}^{\prime}\left(a_{1}+A_{1} x_{2}\right)$

$+p_{2}^{\prime}\left(a_{2}+A_{2} x_{2}\right)-\frac{1}{4}\left(p_{1}^{\prime} B_{1}+p_{2}^{\prime} B_{2}\right) R^{-1}\left(B_{1}^{\prime} p_{1}+B_{2}^{\prime} p_{2}\right)$

where $p_{1}$ and $\epsilon p_{2}$ play the role of the costate variables . Denote $S_{i j}=\frac{1}{2} B_{i} R^{-1} B_{j}^{\prime}$. The corresponding Hamiltonian system has the form:

$$
\begin{gathered}
\dot{x}_{1}=f_{1}\left(x_{1}, p_{1}, x_{2}, p_{2}\right), \quad \dot{p}_{1}=f_{2}\left(x_{1}, p_{1}, x_{2}, p_{2}\right) \\
\epsilon \dot{x}_{2}=A_{2} x_{2}-S_{22} p_{2}+f_{3}\left(x_{1}, p_{1}\right), \\
\epsilon \dot{p}_{2}=-Q x_{2}-A_{2}^{\prime} p_{2}+f_{4}\left(x_{1}, p_{1}\right)
\end{gathered}
$$

where

$$
\begin{aligned}
& f_{1}=a_{1}+A_{1} x_{2}-S_{11} p_{1}-S_{12} p_{2}, f_{2}=-\nabla_{x_{1}} \mathcal{H} \\
& f_{3}=a_{2}-S_{21} p_{1}, \quad f_{4}=-A_{1}^{\prime} p_{1}-s .
\end{aligned}
$$

For each $\epsilon>0$, if $V(x) \geq 0$ is a solution of the Hamilton-J acobi equation

$$
\begin{gathered}
{\left[r+s^{\prime} x_{1}+x_{2}^{\prime} Q x_{2}\right]+V_{x_{1}}\left(a_{1}+A_{1} x_{2}\right)+\frac{1}{\epsilon} V_{x_{2}}\left(a_{2}+A_{2} x_{2}\right)} \\
-\frac{1}{4}\left(V_{x_{1}} B_{1}+\frac{1}{\epsilon} V_{x_{2}} B_{2}\right) R^{-1}\left(B_{1}^{\prime} V_{x_{1}}+\frac{1}{\epsilon} B_{2}^{\prime} V_{x_{2}}\right)=0
\end{gathered}
$$

where $\left(V_{x_{1}}, V_{x_{2}}\right)$ denotes the Jacobian matrix of $V$, such that $V(0)=0$ and the controller given by

$$
u=-\frac{1}{2} R^{-1}\left[B_{1}^{\prime}, \epsilon^{-1} B_{2}^{\prime}\right] V_{x}^{\prime}
$$

is stabilizing, then this controller is the minimizing one. The latter is equivalent to the existence of the invariant manifold of (2.3)

$$
p_{1}=Z_{1}\left(x_{1}, x_{2}\right), \quad p_{2}=Z_{2}\left(x_{1}, x_{2}\right),
$$

where

$$
V_{x_{1}}=Z_{1}^{\prime}, \quad V_{x_{2}}=\epsilon Z_{2}^{\prime}
$$

with asymptotically stable flow

$$
\begin{aligned}
& \dot{x}_{1}=a_{1}+A_{1} x_{2}-S_{11} Z_{1}-S_{12} Z_{2} \\
& \epsilon \dot{x}_{2}=a_{2}+A_{2} x_{2}-S_{21} Z_{1}-S_{22} Z_{2}
\end{aligned}
$$

and such that $V \geq 0$ and $V(0)=0$ (that implies $V_{x}(0)=0$ ). Note that the manifold (2.7) is not necessarily the stable manifold of the Hamiltonian system (2.3) because (2.9) needs not to be exponentially stable [5]. Also, asymptotic stability of (2.9) implies $V \geq 0$.

\section{Main results}

For each $x_{1} \in \mathbf{R}^{\mathbf{n}_{1}}$ consider the fast linear subproblem

$$
\begin{gathered}
\dot{x}_{2}=A_{2}\left(x_{1}\right) x_{2}+B_{2}\left(x_{1}\right) u, \\
J=\int_{0}^{\infty}\left[x_{2}^{\prime} Q\left(x_{1}\right) x_{2}+u^{\prime} R\left(x_{1}\right) u\right] d t
\end{gathered}
$$

and the corresponding algebraic Riccati equation

$$
A_{2}^{\prime} M+M A_{2}+Q-M S_{22} M=0 \text {. }
$$

We assume further 
A1. (3.2) has a positive definite symmetric solution $M\left(x_{1}\right)$, continuous on $x_{1} \in \mathbf{R}^{\mathbf{n}_{\mathbf{1}}}$, such that for each $x_{1} \in \mathbf{R}^{\mathbf{n}_{1}}$ the matrix $A_{2}-B_{2} R^{-1} B_{2}^{\prime} M$ is Hurwitz.

Note that if the pair $\left\{A_{2}\left(x_{1}\right), B_{2}\left(x_{1}\right)\right\}$ is controllable uniformly in $x_{1}$, then A1 holds [6]. Consider the matrix

$$
R_{22}\left(x_{1}\right)=\left(\begin{array}{cc}
A_{2} & -S_{22} \\
-Q & -A_{2}^{\prime}
\end{array}\right)
$$

Under A1 $R_{22}$ possesses the following property: it has $n_{2}$ stable eigenvalues $\lambda, \operatorname{Re} \lambda<-\alpha<0$, and $n_{2}$ unstable ones $\lambda, \operatorname{Re} \lambda>\alpha$ for all $\left|x_{1}\right| \leq m$. Then for any $m>0$ there exists $\epsilon_{m}>0$, such that for all $\epsilon \in\left(0, \epsilon_{m}\right]$ and $\left|x_{1}\right|+\left|p_{1}\right|<m$ the system (2.3) has the slow manifold [6], [9]

$$
\left(\begin{array}{l}
x_{2} \\
p_{2}
\end{array}\right)=\left(\begin{array}{l}
L_{3}^{*}\left(x_{1}, p_{1}, \epsilon\right) \\
L_{4}^{*}\left(x_{1}, p_{1}, \epsilon\right)
\end{array}\right)=L^{*}\left(x_{1}, p_{1}, \epsilon\right)
$$

The subscripts of $L^{*}$ correspond to the third and the fourth variables in the system of (2.3). To avoid cumbersome notation we shall omit $\epsilon$ argument in the functions below.

Setting (3.4) into (2.3a,b) and substituting $v_{1}$ and $w_{1}$ for $x_{1}$ and $p_{1}$ respectively, we get the $2 n_{1}$ dimensional system for the flow on the slow manifold:

$$
\begin{aligned}
& \dot{v}_{1}=f_{1}\left[v_{1}, w_{1}, L_{3}^{*}\left(v_{1}, w_{1}\right), L_{4}^{*}\left(v_{1}, w_{1}\right)\right], \\
& \dot{w}_{1}=f_{2}\left[v_{1}, w_{1}, L_{3}^{*}\left(v_{1}, w_{1}\right), L_{4}^{*}\left(v_{1}, w_{1}\right)\right] .
\end{aligned}
$$

The function $L^{*}$ can be found in the form of expansion

$$
L^{*}\left(x_{1}, p_{1}, \epsilon\right)=\sum_{j=0}^{q} \epsilon^{j} l_{j}^{*}\left(x_{1}, p_{1}\right)+O\left(\epsilon^{q+1}\right) .
$$

The terms of (3.6) can be determined from the equation

$\epsilon \frac{\partial L^{*}}{\partial x_{1}} f_{1}+\epsilon \frac{\partial L^{*}}{\partial p} f_{2}=\left(\begin{array}{c}A_{2} L_{3}^{*}-S_{22} L_{4}^{*}+f_{3}\left(x_{1}, p_{1}\right) \\ -Q L_{3}^{*}-A_{2}^{\prime} L_{4}^{*}+f_{4}\left(x_{1}, p_{1}\right)\end{array}\right)$,

where $f_{i}=f_{i}\left(x_{1}, p_{1}, L_{3}^{*}, L_{4}^{*}\right), i=1,2$, by algebraic operations. Thus, $l_{0}^{*}=-R_{22}^{-1} f_{0}$, where $f_{0}=$ $\operatorname{col}\left\{f_{3}, f_{4}\right\}$. Note that (3.7) can be derived by differentiating on $t$ of (3.4), where $x_{1}=v_{1}(t), p_{1}=$ $w_{1}(t), x_{2}=x_{2}(t), p_{2}=p_{2}(t)$, and by substituting for $\dot{v}_{1}$ and $\dot{w}_{1}$ the right sides of (3.5).

Consider the slow system (3.5). Denote by $\Omega_{m_{i}}=$ $\left\{x_{i} \in \mathbf{R}^{\mathbf{n}_{\mathbf{i}}}:\left|x_{i}\right|<m_{i}\right\}, i=1,2$. Our next assumption is

A2. There exist $m_{1}>0$ and $\epsilon_{1}>0$ such that for all $\epsilon \in\left(0, \epsilon_{1}\right]$ and $v_{1} \in \Omega_{2 m_{1}}$ the system (3.5) possesses the invariant manifold

$$
w_{1}=N\left(v_{1}\right)
$$

where the function $N=N\left(v_{1}, \epsilon\right)$ is continuous on both arguments and uniformly bounded together with its first derivative on $v_{1}$, and $N(0)=0$.

The restriction of (3.5) to (3.8) is governed by the $n_{1}$ - dimensional system

$$
\dot{v}_{1}=F_{1}\left(v_{1}\right),
$$

where

$F_{i}\left(v_{1}\right)=f_{i}\left[v_{1}, N\left(v_{1}\right), L_{3}^{*}\left(v_{1}, N\left(v_{1}\right)\right), L_{4}^{*}\left(v_{1}, N\left(v_{1}\right)\right)\right]$

and $i=1$. Additionally we assume

A3. For all $\epsilon \in\left(0, \epsilon_{1}\right]$ equation (3.9) is asymptotically stable.

It can be shown that $\mathrm{A} 2$ and $\mathrm{A} 3$ are necessary conditions for the existence of the invariant manifold (2.7) with asymptotically stable flow (2.9). Note that the stable solutions of (2.3) are exponentially approaching to the solutions of the slow manifold (3.4) [9]. Under A1-A3 we shall construct the invariant manifold (2.7) with the stable flow by means of the slow submanifold (3.8) and a fast manifold of an auxiliary system. To get the latter system let us introduce the following change of variables:

$$
\begin{gathered}
\left(\begin{array}{c}
v_{2} \\
\bar{p}_{2}
\end{array}\right)=\left(\begin{array}{l}
x_{2} \\
p_{2}
\end{array}\right)-L^{*}\left(x_{1}, p_{1}\right), \\
\left(\begin{array}{l}
\bar{x}_{1} \\
\bar{p}_{1}
\end{array}\right)=\left(\begin{array}{l}
x_{1} \\
p_{1}
\end{array}\right)-\left(\begin{array}{c}
v_{1} \\
w_{1}
\end{array}\right),
\end{gathered}
$$


where $v_{1}$ and $w_{1}$ satisfy (3.5). For the new variables we get the system that possesses for all small $\epsilon$ the fast (stable) manifold for $\left|v_{2}\right|<m^{\prime}[9]$

$$
\left(\begin{array}{l}
\bar{x}_{1} \\
\bar{p}_{1}
\end{array}\right)=\left(\begin{array}{c}
\epsilon L_{1}^{+}\left(v_{1}, w_{1}, v_{2}\right) \\
\epsilon L_{2}^{+}\left(v_{1}, w_{1}, v_{2}\right)
\end{array}\right), \quad \bar{p}_{2}=L_{4}^{+}\left(v_{1}, w_{1}, v_{2}\right),
$$

where $L_{4}^{+}=M\left(v_{1}\right) v_{2}+O(\epsilon)$. The solutions lying on this manifold are rapidly exponentially decaying as $t \rightarrow \infty$. Substituting (3.8) and (3.12) into (3.11) we get the algebraic equations for $Z_{1}$ and $Z_{2}$ determination:

$$
\begin{gathered}
x_{1}=v_{1}+\epsilon L_{1}^{+}\left[v_{1}, N\left(v_{1}\right), v_{2}\right] \\
x_{2}=v_{2}+L_{3}^{*}\left[x_{1}, N\left(v_{1}\right)+\epsilon L_{2}^{+}\left(v_{1}, N\left(v_{1}\right), v_{2}\right)\right]
\end{gathered}
$$

and

$$
\begin{gathered}
p_{1}=N\left(v_{1}\right)+\epsilon L_{2}^{+}\left[v_{1}, N\left(v_{1}\right), v_{2}\right], \\
p_{2}=L_{4}^{*}\left[x_{1}, N\left(v_{1}\right)+\epsilon L_{2}^{+}\left(v_{1}, N\left(v_{1}\right), v_{2}\right)\right] \\
+L_{4}^{+}\left(v_{1}, N\left(v_{1}\right), v_{2}\right) .
\end{gathered}
$$

Consider (3.14) as the system with respect to $v_{1}$ and $v_{2}$. Using the contraction principle argument, one can prove that there exists $\epsilon_{2}$ such that for $\epsilon \epsilon$ $\left(0, \epsilon_{2}\right]$, the system (3.14) has a unique solution on $\Omega_{m_{2}} \times \Omega_{m_{2}}$

$$
v_{1}=V_{1}\left(x_{1}, x_{2}\right)=x_{1}+\epsilon \bar{V}_{1}\left(x_{1}, x_{2}\right),
$$

$v_{2}=V_{2}\left(x_{1}, x_{2}\right)=x_{2}-L_{3}^{*}\left(x_{1}, N\left(x_{1}\right)\right)+\epsilon \bar{V}_{2}\left(x_{1}, x_{2}\right)$

where the functions $\bar{V}_{1}$ and $\bar{V}_{2}$ are Lipschitzian on $x_{1}$ and $x_{2}$, they vanish at $\left(x_{1}, x_{2}\right)=0$, and satisfy the inequalities $\epsilon_{2}\left|\bar{V}_{1}\right| \leq m_{1}, \epsilon_{2}\left|\bar{V}_{2}\right| \leq m^{\prime} / 2$. Further, applying the implicit function theorem one can show that $V_{1}$ and $V_{2}$ are continuously differentiable on $x_{1}$ and $x_{2}$. Substituting (3.15) into (3.14a) and (3.14b) we get $(2.7)$, where

$$
\begin{aligned}
& Z_{1}=N\left(V_{1}\right)+\epsilon L_{2}^{+}\left[V_{1}, N\left(V_{1}\right), V_{2}\right] \\
& Z_{2}=L_{4}^{*}\left[x_{1}, N\left(V_{1}\right)+\epsilon L_{2}^{+}\left(V_{1}, N\left(V_{1}\right), V_{2}\right)\right]
\end{aligned}
$$

$$
+L_{4}^{+}\left(V_{1}, N\left(V_{1}\right), V_{2}\right) \text {. }
$$

Theorem 2. Under A1-A3 for any $m_{2}>0$ there exists $\epsilon_{2}>0$ such that for all $\epsilon \in\left(0, \epsilon_{2}\right]$

(i) the $\left(2 n_{1}+2 n_{2}\right)$-dimensional Hamiltonian system (2.3) has the invariant on $\Omega_{m_{1}} \times \Omega_{m_{2}}$ manifold (2.7) with (2.9) asymptotically stable, where continuously differentiable on $x_{1}$ and $x_{2}$ functions $Z_{1}$ and $Z_{2}$ are defined by formula (3.16) from the algebraic systems of (3.13) and (3.14);

(ii) there exists a $C^{2}$ function $V: \Omega_{m_{1}} \times \Omega_{m_{2}} \rightarrow \mathbf{R}$, satisfying the Hamilton-Jacobi equation (2.5).

To get asymptotic approximation of optimal feedback, optimal trajectory we assume

A4. For small enough $\epsilon$ and $\left|v_{1}\right| \leq 2 m_{1}$ the function $N$ can be represented in the form:

$$
N\left(v_{1}, \epsilon\right)=\sum_{j=0}^{q} \epsilon^{j} N_{j}\left(v_{1}\right)+O\left(\epsilon^{q+1}\right) .
$$

Under A4 $L^{+}$can be found in the form of asymptotic expansion. Then from (3.20) we obtain

$$
Z_{i}=\sum_{j=0}^{q} \epsilon^{j} Z_{i j}\left(x_{1}, x_{2}, \epsilon\right)+O\left(\epsilon^{q+1}\right), \quad i=1,2,
$$

Substituting (5.8) into (2.6) we get the following $O\left(\epsilon^{q+1}\right)$-approximation to the optimal controller :

$u=u_{q}+O\left(\epsilon^{q+1}\right), \quad u_{q}=-\sum_{k=1}^{2} \sum_{j=0}^{q} \epsilon^{j} B_{k}^{\prime} Z_{k j}\left(x_{1}, x_{2}, \epsilon\right)$.

Finally substituting expansions of $v_{1}, v_{2}, L^{+}$and $L^{*}$ into (3.13) and (3.14) and expanding righthand sides of the resulting equations in the powers of $\epsilon$ we find the following approximations:

$$
\begin{aligned}
& x(t)=\sum_{i=0}^{q} \epsilon^{i} x^{(i)}(t)+\sum_{i=0}^{q} \epsilon^{i} \Pi_{1}^{(i)}(\tau)+\epsilon^{q+1} R_{1 q}(t, \epsilon), \\
& u(t)=\sum_{i=0}^{q} \epsilon^{i} u^{(i)}(t)+\sum_{i=0}^{q} \epsilon^{i} \Pi_{2}^{(i)}(\tau)+\epsilon^{q+1} R_{2 q}(t, \epsilon),
\end{aligned}
$$

where $\Pi_{1}$ and $\Pi_{2}$ are boundary layer terms exponentially decaying when $\tau \rightarrow \infty$, remainders $R_{1 q}$ and $R_{2 q}$ are uniformly bounded for $t \in[0, T]$. 


\section{Conclusions}

We have developed a geometric approach to singularly perturbed optimal control problem, nonlinear on the slow state variables. We have got the exact decomposition of the slow-fast invariant manifold of the Hamiltonian system into the reduced-order slow manifold and a fast manifold. As a result, sufficient conditions for the solvability of the optimal control problem in terms of the slow manifold have been obtained. Also, an asymptotic expansion of the optimal controller have been constructed by solving partial differential equation:s, depending only on the slow variables. In the same time we have obtained decomposition of the Hamiltonian system to the slow and fast subsystems. This leads to asymptotic approximation to optimal trajectory and open-loop control. The results are valid on the domains containing large values of the fast variables.

\section{References}

[1] Bensoussan A. Singular perturbations for deterministic control problems, in: Kokotovic P., Bensoussan A. and Blankenship G., Eds., Singular perturbations and Asymptotic Analysis in Control Systems, Lect. Notes in Control and Information Sciences, No 90, Springer-Verlag, Berlin, 1987. pp. 9-170.

[2] Carr J. Applicaitions of centre manifold theory. Springer-Verlag, New York, 1981.

[3] Chow J.H. and Kokotovic P.V. Near-optimal feedback stabilization of a class of nonlinear singularly perturbed systems. SIAM J. Control Optim. 16, pp.756-770, 1978.

[4] Fridman E., "Near-optimal $H_{\infty}$ control of linear singularly perturbed systems", IEEE Trans. on Automat. Contr., AC-41, pp. 236-240, 1996.

[5] Isidori A. and Astolfi A. Disturbance attenuation and $H^{\infty}$-control via measurement feedback in nonlinear systems. IEEE Trans. Automat. Contr., 37, No 9, pp.1283-1293, 1992.

[6] Kokotovic P., Khalil H. and O'Reilly J. Singular Perturbation Methods in Control: Analysis and Design. New York, Academic Press, 1986.

[7] Van der Schaft A. On a state space approach to nonlinear $H_{\infty}$ control. Systems and Control Letters, No 16, pp. 1-8, 1991.

[8] R. O'Malley. Introduction to Singular Perturbations ( Academic Press, New York, 1974).

[9] Sobolev V. Integral manifolds and decomposition of singularly perturbed systems. Systems and Control Letters 4 , pp.169-179, 1984.

[10] W-C. Su, Z. Gajic and X. Shen. The exact slow-fast decomposition of the algebraic Riccati equation of singularly perturbed systems. IEEE Trans. Automat. Contr., AC-37, no. 9, pp. 1456-1459, 1992. 